\title{
Managed Care - ein überzeugendes Konzept
}

\author{
D. Flach
}

\section{* Teilnehmer des Workshops in Scuol waren: Mike Bader (Helsana Versicherungen AG, Versorgungs- management), Ueli Bodmer (Igomed Thun, med-swiss.net), Axel Buchli (SGAM), Roman Buff (Thurcare), Wolfgang Czerwenka (Argomed, Arbeitsgruppe quali- med-net von med-swiss.net), Thomas Eugster (Wintimed), Max- Albrecht Fischer (med-swiss.net), Daniel Flach (Hausärzteverein Biel-Seeland), Hugo Gmür (Kalt- brunn/Linth), Stefan Henzi (mednetbern), Rainer Hurni (zmed Zürich), Urs Keller (Pizol Care), Stefan Koch (Argomed/Freiamt), René Kühne (Helsana Versicherun- gen AG, Versorgungsmanage- ment), Marcel Marolf (Novartis Pharma Schweiz AG), Alfred Rösli (hawa Winterthur-Andelfingen), Markus Sturzenegger (hawa Winterthur-Andelfingen), Ruedi Wartmann (Novartis Pharma Schweiz AG), Andreas Weber (Medix Management AG), Hansueli Zürcher (hap Hausärzte Pfannenstiel).}

Der Workshop wurde gesponsert von der Firma Novartis Pharma Schweiz AG und moderiert von Dr. H. J. Schlegel.

** Zur besseren Lesbarkeit wurde jeweils die männliche Form verwendet, wobei Ärztinnen und Patientinnen miteingeschlossen sind.

\section{Managed Care - eine Idee setzt sich durch}

Bereits zum 7. Mal trafen sich in Scuol Vertreter von Managed-Care-Organisationen der deutschen Schweiz zu einem «Zukunftsworkshop»* Nebst einer Standortbestimmung wurden Erfahrungen mit Projekten ausgetauscht und Ziele für die Managed-Care-Bewegung definiert.

Es zeigte sich, dass Managed-Care-Modelle ein funktionierendes Konzept für die primär regionale, aber auch überregionale medizinische Versorgung darstellen. Wo Managed-Care-Versorgungsmodelle seit Jahren bestehen, haben sich nachweislich die Prozesse der Patientenführung verbessert und das Qualitätsbewusstsein der beteiligten Ärzte ${ }^{* *}$ ist wesentlich gestiegen. Die Förderung der Qualität der Patientenversorgung mittels eines integrativen Patientenmanagements gehört denn auch zu den primären Zielen von Managed-Care-Systemen. In solchen Systemen bietet sich aber auch eine einmalige Gelegenheit, parallel zur Qualitätsüberwachung die Kosten des Behandlungsprozesses zu analysieren und zu kontrollieren.

Der Aufbau und auch die Führung von Managed-Care-Systemen und -Netzwerken bedingt einen gewissen Pioniergeist und ist mit einem nicht unwesentlichen Einsatz von personellen und auch finanziellen Ressourcen verbunden. Der Gewinn aus der Arbeit ist aber bedeutend.

Dies zeigen unter anderem die an der Tagung vorgestellten Projektarbeiten, von denen einige untenstehend kurz skizziert sind. Eine Übersicht aller vorgestellten Projekte und Arbeiten findet sich in der Tabelle 1.

Sowohl die Patienten wie auch die beteiligten Ärzte profitieren von Managed Care. Den Patienten kommt insbesondere die verbesserte Qualität durch die definierten und optimierten Prozessabläufe zugute. Die Ärzte gewinnen durch die Vernetzung an gemeinsamem Profil im Gesundheitswesen und erhalten ein Instrument, um der persönlichen und zunehmend auch ökonomischen Isolierung in der Einzelpraxis wirksam zu begegnen.

Diese positiven Aspekte bewegen immer mehr Ärzte, sich bestehenden Managed-CareModellen anzuschliessen oder motiviert Ärzte- gruppierungen, ein neues lokales Netzwerk nach Managed-Care-Kriterien aufzubauen.

Es bleibt zu erwähnen, dass auch die Leistungsträger mehr und mehr die positiven Aspekte dieser Arbeit erkennen und sich unter anderem durch die Teilnahme am Zukunftsworkshop konstruktiv daran beteiligen. Die Präsentation der Nutzwertanalyse von Ärztenetzwerken durch die Managed-Care-Verantwortlichen der Helsana zeigt, dass in diesem Falle nicht ausschliesslich nur Kostenkriterien das Handeln der Versicherer zeichnet, sondern dass auch Qualitätsaspekte wie Struktur-, Ergebnis- und insbesondere Prozessqualität von wesentlicher Bedeutung sind.

\section{Tabelle 1}

In Scuol vorgestellte Projekte des Jahres 2004. Eine vollständige Übersicht findet sich unter www.forummanaged care.ch $\rightarrow$ Publikationen $\rightarrow$ Präsentationen.

Dr. med. Hansueli Zürcher, hap Hausärzte Pfannenstiel ZH; Dr. med. Urs Keller, Pizol Care, Wangs: Healthcare System in Transition: Zusammenfassung wesentlicher Voten vom Disease Management Congress 2004 in Boston, USA, mit der Frage nach deren Bedeutung für schweizerische Verhältnisse

- Dr. med. Wolfgang Czerwenka, Arbeitsgruppe quali-med-net, med-swiss.net: Qualitätsindikatoren für Ärztenetzwerke

Dr. med. Max-Albrecht Fischer, med-swiss.net: Vision 08: Netzwerke - von der Vision zur Realität

- Dr. med. Markus Sturzenegger, hawa WinterthurAndelfingen: Quick Psycho Diagnostic Panel

- Dr. med. Thomas Eugster, Wintimed, Winterthur: Generikum-Projekt bei Langzeitmedikation

- Dr. med. Thomas Eugster, Wintimed, Winterthur: Nationaler Grippeimpftag bei Wintimed: Erfahrungen mit verbilligter Impfung

- Dr. med. Stefan Koch, Wohlen: Neue Netzwerkentwicklung im Freiamt

- Dr. med. Mike Bader, Helsana Versicherungen AG, Versorgungsmanagement: Nutzwertanalyse von Ärztenetzwerken

- Dr. med. Andreas Weber, medix Management AG: Virtueller Qualitätszirkel e-QZ

Dr. med. Ueli Bodmer, Igomed, Thun: Rx-NET, ÄrzteApotheken-Netzwerk

Dr. med. Urs Keller, Pizol Care, Wangs: Integrierte Versorgung - Vereinbarungen mit lokalen Spitälern, der Spitex sowie den Physiotherapeuten 


\section{Managed-Care-Projekte des Jahres 2004}

Allen Projekten gemeinsam ist das Bestreben, die Qualität der eigenen ärztlichen Arbeit zu analysieren und zum Wohle des Patienten zu verbessern. Eine optimierte Kostenkontrolle kann Inhalt eines Projektes sein. Sie ist aber nicht, wie bereits erwähnt, das ausschliessliche und primäre Ziel von Managed Care, obwohl dies immer noch einer weitverbreiteten Meinung entspricht.

Im folgenden seien einige Projekte kurz dargestellt.

\section{Qualitätsindikatoren für Ärztenetzwerke}

Eine von med-swiss.net, der Dachorganisation der Ärztenetzwerke, eingesetzte Arbeitsgruppe (quali-med-net) formulierte Qualitätsindikatoren für Netzwerke. Es ergaben sich klare Definitionen hinsichtlich der Organisation, der internen und externen Zusammenarbeit, dem Prozessmanagement, der Kommunikation sowie des Outcomes von Netzwerken. Die Indikatoren sollen die Entwicklung einer effizienten medizinischen Versorgung unterstützen und fördern und die beteiligten Ärztenetzwerke für die Qualitätsarbeit sensibilisieren im Wissen darum, dass wer sich um Qualität bemüht, nachweislich seine Qualität verbessert.

\section{Vision 08: Netzwerke - von der Vision zur Realität}

Ebenfalls eine Arbeitsgruppe von med-swiss.net erarbeitete Grundsätzliches zu Entwicklung und Definition von Ärztenetzwerken. Solche Netzwerke werden definiert als Organisationen, die von eigenständigen Leistungserbringern gebildet werden und die der Gesundheitsversorgung dienen durch ein verbindliches Zusammenwirken von formulierten Behandlungsprozessen [1]. Dabei wird eine gemeinsame Betreuungskultur und eine Unternehmensstruktur angestrebt. Es wurden die Qualitätsentwicklung, die Budgetmitverantwortung sowie ein gerechter Risikoausgleich als zu erreichende Ziele erarbeitet. Die fehlende Risikodetermination und ein entsprechender Ausgleich werden heute als Schwachpunkte angesehen. Auf der Basis des Thurgauer Morbiditätsindikators sollen weitere Schritte in dieser Richtung geplant werden. Im weiteren wurde festgehalten, dass Netzwerke nicht primär eine volle Budgetverantwortung, sondern Mitverantwortung anstreben.

\section{Generika bei Langzeitmedikation}

In einem lokalen Projekt wurde festgestellt, dass bei 146 von 1168 evaluierten Patienten eine Umstellung auf ein Generikum möglich war. Unter anderem hatte bereits ein Viertel der Patienten mit Langzeitmedikation ( $n=97$ ) ein Generikum in der Medikamentenliste. Bei noch mehr Patienten (145) bestand eine Medikation ohne Substitutionsmöglichkeit. Bei den meisten Patienten, bei denen eine Umstellung auf ein Generikum erfolgte, wurde dieses beibehalten. Bei immerhin 12\% musste infolge subjektiver Unverträglichkeit wieder auf das Originalpräparat zurückgewechselt werden.

Die teilnehmenden Ärzte profitierten von einer Sensibilisierung hinsichtlich ihrer Verordnungsqualität. Die Akzeptanz der Generika unter den Patienten war generell gross.

\section{Neue Netzwerkentwicklungen}

Angesichts der obenerwähnten Vorteile von Ärztenetzwerken erstaunt es fast niemanden mehr, dass auch 2004 wieder mehrere lokale und überregionale Gruppierungen entstanden sind mit dem Ziel, Managed Care im Praxisalltag einfliessen zu lassen. Präsentiert haben sich in Scuol die neuen Netzwerke Freiamt AG, mednet Bern und Kaltbrunn/Linth.

Um den damit verbundenen Problemen, insbesondere hinsichtlich personeller und zeitlicher Ressourcen, zu begegnen, wird eine vermehrte Professionalisierung der Netzwerke angestrebt.

\section{Managed Care - im Jahre 2005}

Die getätigten Erfahrungen in den vergangenen Jahren haben klar gezeigt, dass Managed Care und Ärztenetzwerke überzeugende Konzepte sind, die sich klar etabliert haben und in Zukunft eine wesentliche Rolle bei der Gestaltung unseres Gesundheitswesens übernehmen werden. Die Kostenproblematik zwingt alle Beteiligten im Rahmen der Ressourcenallokation zu einer besseren Koordination der Behandlungsprozesse. Mit den Ärztenetzwerken haben wir hierfür ein effizientes Instrument zur Hand. Es gilt nun, Managed Care sowohl in der Standesarbeit wie auch politisch besser zu verankern. Mittels vermehrter Öffentlichkeitsarbeit soll die Wichtigkeit der Grundversorgung im Behandlungsprozess betont und die Notwendigkeit eines attraktiven Berufsumfeldes zur Nachwuchssicherung aufgezeigt werden.

\section{Literatur}

1 Fritschi J. Netzwerke - von der Vision zur Realität. PrimaryCare 2005;5(3):48-9. 